Ann. Génét. Sél. anim., I980, 12 (2), I9I-200.

\title{
Composition anatomique, production d'œufs et efficacité alimentaire de poules pondeuses. Corrélations phénotypiques
}

\author{
P. MFRAT $\left({ }^{*}\right)$, A. BORDAS $\left({ }^{*}\right)$ et F. H. RICARD $(* *)$ \\ (*) Laboratoire de Génétique Factorielle, \\ Centre national de Recherches zootechniques, I.N.R.A., \\ 78350 Jouy-en-Josas, France \\ (**) Station Expérimentale du Magneraud, \\ I.N.R.A., I7700 Surgères, France.
}

\section{Résumé}

Sur des poules pondeuses appartenant à deux populations expérimentales, en 1976 et 1978 , les corrélations phénotypiques ont été estimées entre des variables anatomiques et, d'autre part, la production et plusieurs caractéristiques des œufs, le poids corporel et sa variation, la consommation alimentaire corrigée pour le poids, le gain de poids et la masse totale d'œufs pondus, sur trois périodes de 28 jours entre 8 et I 1 mois d'âge.

Sur l'ensemble des données, les corrélations suivantes apparaissent les plus intéressantes (pour les composants anatomiques rapportés au poids corporel) :

- Les pourcentages de graisse abdominale et de peau sont en corrélation positive avec le poids corporel; le pourcentage de sang, de plumes, d'os et de muscles, le cœur et la partie supérieure du tractus digestif diminuent avec la taille de l'animal.

- La consommation "corrigée " d'aliment présente une corrélation positive avec le poids du plumage, celui du foie et du tractus intestinal. Le poids spécifique du tibia est lié positivement au gain de poids corporel, négativement au nombre et à la masse d'œufs pondus. Cette dernière est en corrélation positive avec le poids de peau et du cœur.

- La graisse abdominale est en forte corrélation positive avec le pourcentage de peau et le poids du foie, négative avec le tractus génital. Le pourcentage d'os du membre inférieur est en corrélation positive avec le poids de plumes, et ce đernier est lié négativement à l'importance des lipides abdominaux. Le poids du cœur varie dans le même sens que celui des muscles du membre inférieur et est lié négativement au poids spécifique du tibia.

L'interprétation et l'intérêt pratique possible de certaines de ces corrélations sont discutés.

\section{Introduction}

De nombreux travaux se rapportent à l'état d'engraissement et à la teneur en lipides des poulets de chair, par exemple WAShBuRn et al., 1976; Dror et al., I977; voir revue par RICARD, I977. Beaucoup moins d'observations ont été faites 
sur les autres composantes anatomiques des carcasses et leurs corrélations mutuelles : RICARD et RoUvIER, I967, I969; RICARD, I972, I974. Quant aux travaux analogues sur les poules pondeuses, ils concernent presque exclusivement, à notre connaissance, l'importance des tissus gras, évaluée directement, ou indirectement d'après l'épaisseur de la peau (GREENBERG, I976) ou le gain de poids des poules adultes (BORDAS et MÉRAT, I977; LECLERCo et al., I977). Ces derniers auteurs mettent ce critère en relation avec l'efficacité alimentaire des pondeuses. Sur d'autres composantes anatomiques et leurs corrélations avec des caractères de production, on peut encore citer HESTER et al., I979, à propos d'une relation entre taille de l'ovaire et fréquence de la ponte d'œufs sans coquille.

Il nous a donc paru intéressant de rechercher les relations de diverses composantes anatomiques des poules pondeuses obtenues par dissection, aussi bien entre elles qu'avec les performances relatives à la production d'œufs et à l'efficacité alimentaire.

\section{Matériel et méthodes}

\section{a. - Matériel animal et conditions d'élevage}

Deux populations ont été utilisées :

- une population expérimentale (" population I ") en ségrégation pour certains gènes connus, en l'occurrence les allèles liés au sexe $\mathrm{S}$ et $s$. La présente étude porte sur deux échantillons de cette population, l'un éclos à l'automne I975, 1'autre à 1'automne I977. Le second échantillon provenait d'une sous-lignée distincte du premier, de poids corporel moyen plus élevé. En I 976, 7I poules adultes, issues de 3 familles de pères étaient utilisées, et en 1978,63 poules issues de 5 pères;

- une population de race Rhode-Island ("population 2 ") subdivisée en deux lignées, dont un échantillon, éclos en janvier I976, est l'objet du présent travail, avec respectivement 32 et 30 poules adultes, issues de 8 pères pour chaque lignée. Ces lignées constituaient la première génération d'une expérience de sélection sur l'efficacité alimentaire des poules adultes (sélection divergente sur la consommation alimentaire " résiduelle " $\mathrm{R}$, définie au $\S$ suivant).

Les poulettes étaient élevées en poussinière au sol jusqu'à 16 semaines d'âge, puis transférées en cages individuelles pour enregistrement des performances de ponte et de la consommation alimentaire sur 3 mois (voir $\S b$ ). Durant la période de ponte, l'aliment, donné sous forme de farine, contenait I 6 p. roo de protéines totales et $2520 \mathrm{Kcal} / \mathrm{kg}$ ( Io,6 MJ) d'énergie métabolisable. Le jour naturel était supplémenté par un éclairage électrique pour obtenir constamment $\mathrm{I} 4 \mathrm{~h}$ de lumière par $24 \mathrm{~h}$. Les dissections étaient faites approximativement à II mois d'âge.

\section{b. - Mesures}

Les mesures concernant la ponte et la consommation alimentaire sont basées sur trois périodes consécutives de 28 jours après le pic de ponte, entre les âges de 8 et II mois. Flles comprennent d'abord les variables suivantes (moyennes des 3 périodes de 28 jours exprimées en g.) :

- poids corporel moyen $(\mathrm{P})$, moyenne des poids en début et en fin de période; 
- variation de poids $(\Delta \mathrm{P})$, du début à la fin de la période;

- masse d'œufs produits (E);

- consommation d'aliment (O).

Une variable dérivée, $R$, est obtenue comme suit : la consommation théorique d'aliment $\mathrm{T}$ est estimée par individu à partir d'une équation de régression multiple incluant $\mathrm{P}, \Delta \mathrm{P}$ et $\mathrm{E}$ (BYERLY, I94I) :

$$
\mathrm{T}=a \mathrm{~F}^{\alpha}+b \Delta \mathrm{P}+c \mathrm{E}
$$

La valeur 0,5 est utilisée pour $\alpha$ (cf. MERAT et BORDA,S, I979). La différence $\mathrm{R}=\mathrm{O}$ - $\mathrm{T}$ est appelée " consommation résiduelle ".

Le nombre d'œufs (N.o.) pondus durant l'ensemble des trois périodes et le poids moyen des œufs (P.o.) basé sur la ponte de deux semaines dans la dernière période de 28 jours sont également notés. Enfin, la hauteur de l'albumen (H.a.) de l'œuf cassé sur une surface plane et l'épaisseur de la coquille (E.c.), moyenne de 2 œufs au cours de la même période, sont enregistrés (respectivement en $\frac{\mathrm{I}}{\mathrm{IO}} \mathrm{mm}$ et $\left.\frac{\mathrm{r}}{\mathrm{I} O \mathrm{O}} \mathrm{mm}\right)$.

Le groupe des variables relatives à la composition anatomique a été obtenu par dissection sur les poules à I I mois d'âge. En I976, ces dissections ont été faites à la Station I.N.R.A. du Magneraud, en I978 à Jouy-en-Josas. Le tableau I contient la définition de ces variables et les symboles qui les désignent dans les tableaux suivants. Les poids d'organes ou tissus sont exprimés en pour cent du poids cor-

\section{TABLEAU I}

Symbole et défnition des veriables obtenues par dissection Symbol and definition of variables connected with body composition

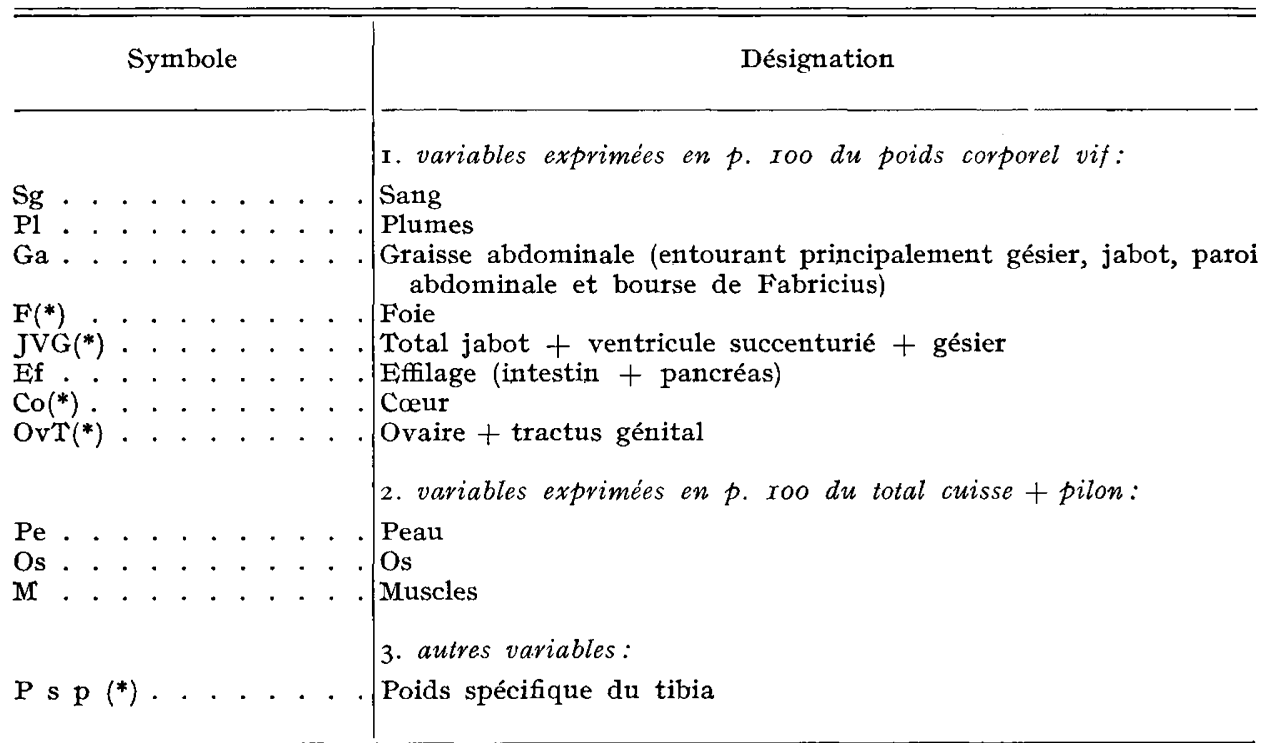

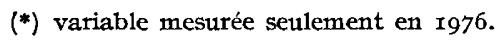


porel avant abattage, à l'exception des poids de peau, os et muscles (p. Ioo du poids total cuisse + pilon) et du poids spécifique du tibia. Une description plus complète de ces mesures est donnée par RICARD et RouviER (I967), RICARD (I972, I974). Nous rappelons seulement que le poids du sang et celui du plumage sont obtenus par différence entre le poids de 1'animal avant et après respectivement saignée ou plumage $\left.{ }^{\mathbf{1}}\right)$. La graisse abdominale est celle entourant une partie des organes digestifs $\left({ }^{2}\right)$. Les segments du tractus digestif (jabot, ventricule succenturié, gésier, et effilage correspondant au tractus intestinal) sont pesés vides.

Seules sont retenues, dans chaque série expérimentale, les poules mesurées pour toutes les variables enregistrées dans cette série.

TABLEAU 2

Valeurs moyennes par série expérimentale mean values for each experimental series

\begin{tabular}{|c|c|c|c|c|c|c|c|c|c|c|}
\hline & \multirow{3}{*}{\multicolumn{4}{|c|}{ Caractère }} & & & \multicolumn{4}{|c|}{ Série expérimentale } \\
\hline & & & & & & & \multicolumn{2}{|c|}{ Population I } & \multicolumn{2}{|c|}{ Population 2 (1976) } \\
\hline & & & & & & & $\begin{array}{c}\text { I976 } \\
(n=7 \mathrm{I})\end{array}$ & $\begin{array}{c}1978 \\
(\mathrm{~N}=63)\end{array}$ & $\begin{array}{c}\mathrm{R}+ \\
(n=32)\end{array}$ & $\underset{(n=30)}{\mathrm{R}-}$ \\
\hline $\begin{array}{l}\mathrm{P} . \cdot \\
\Delta \mathrm{P} . \\
\mathrm{E} . \\
\mathrm{O} . \\
\mathrm{R} . \\
\text { N.o. } \\
\text { P.o. } \\
\text { H.a. } \\
\text { E.c. }\end{array}$ & 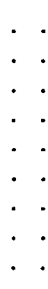 & 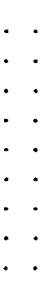 & $\begin{array}{l}\dot{.} \\
\dot{.} \\
\dot{.} \\
\dot{.} \\
\dot{.}\end{array}$ & $\begin{array}{l}\dot{.} \\
\dot{.} \\
\dot{.} \\
\dot{.} \\
\dot{.} \\
\dot{.} \\
\dot{.}\end{array}$ & $\begin{array}{l}\cdot \\
\cdot \\
\cdot \\
\cdot \\
\cdot \\
\cdot \\
\cdot \\
\cdot \\
\cdot\end{array}$ & $\begin{array}{ll}\cdot & \cdot \\
\cdot & . \\
\cdot & . \\
\cdot & . \\
\cdot & . \\
\cdot & . \\
\cdot & . \\
. & . \\
\cdot & .\end{array}$ & $\begin{array}{r}\text { I } 956, \mathrm{I} \\
33,0 \\
\text { I } 043,8 \\
3287,9 \\
0,6 \\
60,0 \\
54, \mathrm{I} \\
64, \mathrm{I} \\
38,7\end{array}$ & $\begin{array}{r}2869,6 \\
60,6 \\
1085,3 \\
3998,0 \\
\quad 14,7 \\
56,9 \\
60,8 \\
70,5 \\
37,4\end{array}$ & $\begin{array}{r}2250,0 \\
-12,7 \\
926,5 \\
3004,3 \\
209,3^{*} \\
50,7 \\
56,3 \\
80,3 \\
36,1\end{array}$ & $\begin{array}{c}2057,5 \\
-2 \mathrm{I}, 2 \\
904,8 \\
26 \mathrm{I} 8, \mathrm{r} \\
-\mathrm{I} 02, \mathrm{I} * \\
44,8 \\
55,5 \\
84,3 \\
36,4\end{array}$ \\
\hline $\begin{array}{l}\mathrm{Sg} . \\
\mathrm{Pl} . \\
\mathrm{Ga} . \\
\mathrm{F} . \\
\text { JVG } \\
\text { Ef } \\
\text { Co. } \\
\text { OvT. }\end{array}$ & $\begin{array}{l}\dot{.} \\
\dot{\cdot} \\
\dot{.} \\
\dot{.} \\
\dot{.} \\
.\end{array}$ & 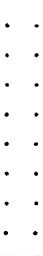 & $\begin{array}{l}\dot{.} \\
\dot{.} \\
\dot{.} \\
\dot{.} \\
\dot{.}\end{array}$ & $\begin{array}{l}\cdot \\
\dot{.} \\
. \\
. \\
. \\
. \\
. \\
.\end{array}$ & $\begin{array}{l}\cdot \\
\cdot \\
\cdot \\
\cdot \\
\cdot \\
\cdot \\
\cdot \\
\cdot\end{array}$ & $\begin{array}{cc}\cdot & \cdot \\
\cdot & \cdot \\
\cdot & \cdot \\
\cdot & \cdot \\
\cdot & \cdot \\
\cdot & \cdot \\
\cdot & \cdot \\
\cdot & \cdot\end{array}$ & $\begin{array}{l}4, \mathrm{I} \\
6,6 \\
3,9 \\
\mathrm{I}, 4 \\
2, \mathrm{I} \\
4,2 \\
0,4 \mathrm{I} \\
5,7\end{array}$ & $\begin{array}{l}3,4 \\
4,6 \\
7,9 \\
- \\
3,9 \\
-\end{array}$ & $\begin{array}{l}3,4 \\
6,2 \\
6,3 \\
\mathrm{I}, 5 \\
\mathrm{I}, 9 \\
3,7 \\
0,40 \\
4, \mathrm{I}\end{array}$ & $\begin{array}{l}3,2 \\
6,8 \\
5,4 \\
1,3 \\
2,0 \\
3,7 \\
0,39 \\
4,2\end{array}$ \\
\hline $\begin{array}{l}\mathrm{Pe} . \\
\text { Os } \\
\mathrm{M} \\
\text { Psp. }\end{array}$ & $\begin{array}{l}\dot{.} \\
\dot{.}\end{array}$ & $\begin{array}{ll}\cdot & \cdot \\
\cdot & . \\
. & . \\
\cdot & .\end{array}$ & $\dot{\dot{y}}$ & $\dot{.} \cdot$ & $\dot{.}$ & $\begin{array}{ll}\cdot & . \\
. & . \\
. & . \\
. & .\end{array}$ & $\begin{array}{c}9,9 \\
\text { I I,8 } \\
74, \mathbf{I} \\
\text { I,33 }\end{array}$ & $\begin{array}{r}\text { ro,6 } \\
9,4 \\
76,7 \\
-\end{array}$ & $\begin{array}{c}15,7 \\
9,9 \\
70,7 \\
1,34\end{array}$ & $\begin{array}{l}\text { 1 } 3,9 \\
\text { 10,6 } \\
71,8 \\
\text { I,32 }\end{array}$ \\
\hline
\end{tabular}

(*) Calculé à partir d'une équation commune pour $\mathrm{R}^{+}$et $\mathrm{R}^{-}$.

(i) après trempage en 1976 ; à sec en 1978 .

(2) principalement gésier et bourse de Fabricins, la graisse entourant 1'intestin étant exclue. 


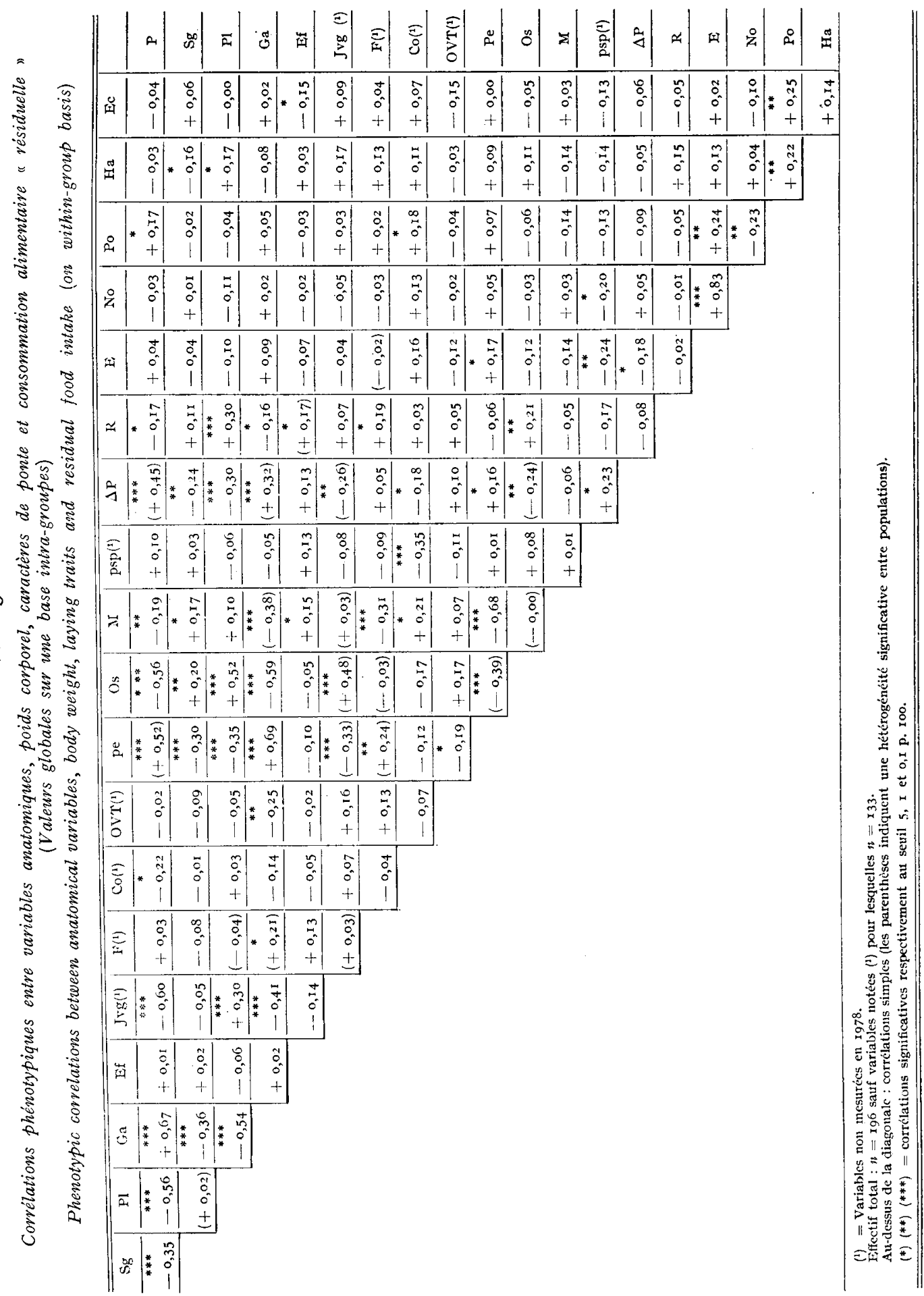




\section{c. - Analyse statistique des données}

Les corrélations phénotypiques sont estimées à l'intérieur de chaque groupe expérimental (population-année), puis regroupées après test de leur homogénéité entre groupes.

Les distributions de fréquences de quelques variables anatomiques (G.a. surtout) s'écartent significativement de la normalité. Ayant vérifié qu'une transformation (racine carrée) de cette dernière variable ne modifiait pas sensiblement les corrélations qui s'y rapportent, nous ne présentons pas ici le détail correspondant.

\section{Résultats}

Les valeurs moyennes, par caractères et par série expérimentale, sont donnée au tableau 2 .

Le tableau 3 présente, dans sa partie supérieure, les corrélations phénotypiques entre tous les caractères, globalement pour l'ensemble des quatre souspopulations.

\section{Discussion}

I. Les valeurs moyennes sont données à titre indicatif. I,e poids corporel nettement différent dans la première population en I976 et I978 correspond, comme il a été signalé plus haut, à l'utilisation de deux sous-populations différant pour ce caractère. On remarque que la sous-population la plus lourde a plus de graisse abdominale et moins de plumes. La seconde population a, en pourcentage, plus de peau, et un tractus génital plus réduit, mais un effet "saison " peut être superposé à l'effet " population ".

2. Plusieurs des corrélations avec le poids corporel méritent attention. Le pourcentage de graisse abdominale et de peau et le gain de poids augmentent avec lui; le pourcentage de sang, de plumes, d'os et de muscles, le cœur et la partie supérieure du tractus digestif (jabot, ventricule succenturié, gésier) diminuent. La corrélation avec la consommation " résiduelle " provient vraisemblablement du hasard de l'échantillonnage, car la définition de cette variable la rend en principe indépendante du poids de l'animal, mais le présent échantillon n'est qu'une partie de la population sur laquelle les coefficients de l'équation de régression multiple permettant de calculer la variable $R$ ont été estimés.

La plupart des corrélations citées sont hautement significatives, mais leur interprétation n'est pas évidente. Ia diminution relative du plumage lorsque le poids augmente pourrait refléter une liaison de cette variable plutôt avec la surface corporelle qu'avec le poids, lié au volume. Par contre, on ne peut invoquer la même raison en ce qui concerne la peau, dont le pourcentage varie dans le même sens que le poids : en l'occurrence, cela paraît correspondre à la liaison peau graisse abdominale et lipides totaux commentée plus loin.

Nous n'avons pas connaissance, d'autre part, de travaux antérieurs concernant les corrélations mentionnées, à l'exception de celle avec le gain de poids (BORDAS et MERAT, I977). 
3. Entre les variables relatives à la production d'œufs et à la consommation alimentaire, les corrélations positives, d'une part de la masse d'œufs avec le nombre et le poids moyen des œufs, de l'autre entre hauteur de l'albumen et poids de l'œuf, sont bien connues et n'ont pas besoin d'être commentées davantage. La corrélation négative entre le poids moyen des œufs et leur nombre, significative au seuil 5 p. Ioo, n'est pas retrouvée dans des échantillons plus nombreux des mêmes populations, non plus que celle, positive, entre consommation résiduelle et hauteur de l'albumen (BORDAS et MERAT, I974, et données non publiées), ni celle entre gain de poids et poids des œufs, mais par contre nous retrouvons sur l'ensemble de nos résultats la corrélation positive observée ici entre poids moyen de 1'œuf et épaisseur de coquille. Quant à la corrélation négative entre gain de poids corporel et masse d'œufs pondue, elle a été signalée antérieurement (Bordas et MÉRAT, I977).

4. Entre ces mêmes variables et les variables obtenues par dissection, plusieurs corrélations positives significatives se rapportent à la consommation résiduelle $(R)$ : avec le poids du plumage, la proportion d'os du nembre inférieur $(P<0,0 I)$, le poids du foie et celui du tractus intestinal ou effilage $(\mathrm{P}<0,05)$, avec une réserve sur cette dernière corrélation dûe à son hétérogénéité entre populations. Avec la graisse abdominale, la corrélation est légèrement négative $(\mathrm{P}<0,05)$. Cependant, toutes les variables ci-dessus ne sont pas indépendantes les unes des autres. Deux d'entre elles, os et graisse abdominale, sont en forte corrélation positive avec le poids de plumes, qui est lui-même la variable la plus liée à $R$; la corrélation partielle de ces deux variables avec $R$ à poids de plumes fixé est respectivement $+0,07$ et 0,00 (non significatif). Par contre, les trois variables " plumes ", "foie " et " effilage " restent chacune significativement liée à $R$ même en fixant les autres variables.

Le gain de poids $(\Delta \mathrm{P})$ présente des corrélations significatives avec diverses variables anatomiques, mais la plupart sont le reflet de la forte corrélation positive qu'il présente avec le poids corporel $\mathrm{P}$ : à poids vif fixé, on vérifie que le gain de poids n'est en corrélation (positive) qu'avec le poids spécifique du tibia. La masse d'œufs ( $\mathbf{E}_{\text {) }}$ est liée significativement et négativement avec cette même variable, positivement avec le poids du cœur et le pourcentage de peau. Le nombre d'œufs ne révèle qu'une corrélation, de même sens que pour la masse d'œufs, avec le poids spécifique du tibia. Le poids moyen des œufs n'est lié qu'avec celui du cœur. Les corrélations significatives relatives à la hauteur de l'albumen et à l'épaisseur de coquille concernent respectivement le plumage, le sang pour la première variable, l'effilage pour la seconde. (On vérifie que ces corrélations subsistent à poids vif fixé).

La plupart de ces corrélations n'ont pas d'interprétation claire pour l'instant. Quelques remarques peuvent être faites. La corrélation positive très significative de la consommation résiduelle $(\mathrm{R})$ avec le poids relatif du plumage à poids corporel fixé est évidemment un phénomène tout à fait distinct de l'augmentation des besoins énergétiques consécutifs à la détérioration du plumage au cours de la ponte (Charles, i976; WAThes, I976; LEEson et Morrison, I978) : ce dernier phénomène devrait logiquement conduire à une corrélation de signe opposé, et de toutes façons il était très peu important dans les cages utilisées ici $\left({ }^{1}\right)$. On peut se

( ${ }^{1}$ On peut aussi noter que, les pour individus à plus grande proportion de plumes, le poids métabolique en proportion du poids vif doit être moindre, entraînant une consommation alimentaire légèrement plus faible que prévu, ce qui devrait avoir pour résultat une petite corrélation négative entre $R$ et le poids des plumes. 
demander par ailleurs si les corrélations de la variable $\mathrm{R}$ avec le poids relatif de certains parties du tractus digestif correspondent à des différences d'efficacité digestive touchant certains nutriments, associés à la taille de ces fractions.

Quant à la corrélation négative de la masse totale et du nombre d'œufs avec le poids spécifique du tibia, il paraît plausible de la rapporter à un effet sur le métabolisme du calcium squelettique associé aux variations d'intensité de ponte. La corrélation du poids moyen des œufs avec le poids du cœur peut faire penser à un effet sur la vitellogenèse de l'intensité, de la circulation sanguine. D'autres éléments seraient bien entendu nécessaires pour confirmer ou infirmer ces hypothèses.

\section{5. - Corrélations des variables anatomiques entre elles}

A certaines de ces variables ne sont attachées qu'une ou deux corrélations significatives. C'est le cas du poids spécifique du tibia (avec le cœur), du cœur (avec les muscles), de 1'ensemble ovaire - tractus génital (avec la graisse abdominale et le pourcentage de peau). La relation entre cœur et poids spécifique d'un os long suggère une répercussion de la circulation sanguine sur le métabolisme du squelette (incluant la moelle osseuse); celle entre poids du cœur et poids des muscles du membre inférieur peut refléter une variation dans le même sens des différents muscles en importance relative. La variation en sens inverse de la graisse abdominale et $\mathrm{du}$ tractus génital ne reflète pas une relation de l'engraissement avec la ponte, chacune des deux premières variables ne présentant pas de corrélation avec cette dernière, ce qui d'ailleurs mérite d'être noté.

Pour les variables " peau ", " os " et " muscles ", limitées à une partie du membre inférieur, il est évident que leurs corrélations deux à deux ne peuvent être que négatives puisque leur somme constitue la quasi totalité de ce membre. D'autre part, parmi ces trois variables, la peau est en forte corrélation positive avec la graisse abdominale, ce qui s'accorde avec les conclusions d'autres auteurs quant à l'existence d'une liaison entre la teneur corporelle en lipides totaux et d'une part l'épaisseur de la peau, de l'autre les lipides abdominaux, chez le poulet en croissance du moins (DELPECH et RICARD, I965; RICARD, I968; RICARD et ROUVIF, I969; GREENBERG, I976; EHINGER, I977). D'autre part, la corrélation positive entre graisse abdominale et foie reflète vraisemblablement un effet commun de l'état d'engraissement sur les dépôts adipeux autour des organes digestifs et l'infiltration de lipides dans le foie. Une corrélation analogue est trouvée par NEILL et al. (I977).

Parmi les autres corrélations notables, deux des plus hautement significatives sont celles, positives, entre le pourcentage d'os dans l'ensemble (cuisse + pilon) et le pourcentage de plumage, et celle, négative, entre plumage et graisse abdominale. Le pourcentage d'os est également lié à celui de l'ensemble jabot-ventricule-gésier, alors que le tractus intestinal (effilage) est associé au pourcentage de muscles. Enfin, le pourcentage de sang présente une corrélation négative avec la peau et la graisse abdominale. Ces corrélations subsistent d'ailleurs à poids corporel fixé; ce n'est pas le cas pour quelques autres, que, pour cette raison, nous ne commentons pas davantage.

\section{6. - Intérêt pratique possible}

Bien entendu, il s'agit ici de corrélations phénotypiques, et 1'estimation des corrélations génétiques peut apporter des éléments complémentaires. D'autre part, certaines des mesures obtenues par dissection sont relativement peu précises, 
comme le pourcentage de sang qui peut dépendre d'une saignée plus ou moins complète, ou le poids de l'ovaire lié à l'état de ponte momentané. Néanmoins, outre l'intérêt physiologique de certaines corrélations observées on peut se poser la question de l'utilité éventuelle de quelques unes de ces mesures comme aides à la sélection sur des caractères d'intérêt économique. Ainsi, la liaison positive entre importance relative du plumage et consommation alimentaire "résiduelle " pourrait peut-être être mise à profit, si l'on disposait de mesures praticables sur le vivant et reflétant la première des deux variables de façon suffisamment satisfaisante, la consommation " résiduelle " étant laborieuse à mesurer directement.

En elles-mêmes, certaines des variables obtenues par dissection des carcasses ont une valeur économique non entièrement négligeable en tant que liées au rendement à l'abattage des poules de réforme : c'est le cas du pourcentage de plumes, d'os et d'effilage. Le fait que ces trois variables soient en corrélation positive avec la consommation alimentaire résiduelle $\mathrm{R}$ montre qu'une sélection pour diminuer cette dernière pourrait se répercuter favorablement sur le rendement à l'abattage, ce qui en renforcerait quelque peu l'intérêt, dans la mesure où les corrélations génétiques se révèleront de même signe.

Notons enfin que 1'ensemble des variables anatomiques relevées ici rend compte d'une fraction non négligeable de la variance des caractères mesurant la production d'œufs et son efficacité. Une corrélation canonique entre six variables du premier groupe (pe. os. m. pl. ga. ef.) et quatre variables du second groupe $(\mathrm{P} ; \Delta \mathrm{P} ; \mathrm{E} ; \mathrm{R})$ atteint la valeur $+0,77$.

Reçu pour publication en aồt 1980.

\section{Remerciements}

Nous remercions M. L. OLLIVIER, Station de Génétique Quantitative et appliquée (C.N.R.Z., 78 Jouy-en-Josas) et $M$. R. RouvieR (Station d'amélioration génétique des animaux, 3i-Castanet) de leurs remarques et critiques très utiles à la lecture de ce manuscrit.

\section{Summary}

\section{Anatomical variables, egg production and feed efficiency of laying hens: phenotypic correlations}

On laying hens from two experimental strains, in 1976 and 1978 , phenotypic correlations were estimated between anatomical variables and egg production, egg traits, body weight, body weight variation, food consumption after correction for body weight, weight gain and total egg mass, over three $28 \mathrm{~d}$. periods between 8 and $\mathrm{I} I$ months of age.

The following correlations appear to be the most interesting (with body constituents expressed in p. I oo of body weight) :

- Per cent of abdominal fat and skin show positive correlations with body weight; $p$. Ioo blood, feathers, bone, muscle, heart and upper part of digestive tract decrease with increasing body size.

- Corrected food intake is in positive correlation with weight of plumage, liver and intestine. Specific gravity of tibio-tarsus is positively associated with body weight gain, negatively with egg number and egg mass. Egg mass shows a positive correlation with p. Ioo skin and heart.

- Abdominal fat has a strong positive correlation with skin and liver weight, negative with genital tract. Per cent bone of the lower limb is in positive correlation with plumage per cent; this last variable shows a negative association with abdominal fat. Heart p. cent varies in the same direction as muscle and is negatively associated with specific gravity of tibio-tarsus.

Possible interpretation and practical bearing of some of these correlations is discussed. 


\section{Références bibliographiques}

Becker W. A., Spencer J. V., Mirosh L. W., Verstrate J. A., i979. Prediction of fat and fat-free live weight in broiler chicken using backskin fat, abdominal fat, and live body weight. Poult. Sci. 58, 835-842.

BORDAS A., MERAT P., I974. Variabilité génétique et corrélations phénotypiques caractérisant la consommation alimentaire de poules pondeuses après correction pour le poids corporel et la ponte. Ann. Génét. Sél. anim. 6, 369-379.

Bordas A., MERAT P., I977. Gain de poids pendant la ponte et sélection sur l'efficacité alimentaire chez la poule. Ann. Génét. Sél. anim. 8, 527-536.

BYERLY T. C., I94I. Feeds and other costs of producing market eggs. Bull. no Ar, Univ. of Maryland Agric. Exp. Sta.

ChARLES D. R., I976. The economic importance of feathering. Gleadthorpe Poultry Booklet, 25-27.

DELPECH P., RICARD F. H., I965. Relation entre les dépôts adipeux viscéraux et les lipides corporels chez le poulet. Ann. Zootech. 14, I81-189.

DROR Y., NIR I., NISTAN Z., 1977. The relative growth of internal organs in light and heavy breeds. Brit. Poult. Sci. 18, 493-496.

EHINGER F., 1977. Beziehungen zwischen Hautfett, gänz-körper und plasma werten bei Broilern Verschiedenen Herkunft. Archiv. für Geflügelkunde 41, 35-37.

GREENBERG H. S., 1976. Relationship between body composition and reproductive performance in the laying hen. Diss. abstr. B 37, 2583-2584.

Hester P. Y., Wilson E. K., Pierson F. W., I979. Organ weights in hens with a high incidence of soft-shelled or shelless egg production. Poult. Sci., 58, 1067 (abstr.).

LECLERCQ B., BLUM J. C., BOYER J. P., I977. Significance and genetic control of body weight changes in the hen during the laying period. Archiv. für Geflügelkunde 41, I2I-124.

LEESON S., MORRISON W. D., I978. Effect of feather cover on feed efficiency in laying birds. Poult. Sci. 57, ro94-1096.

MÉRAT P., BORDAS A., 1979. Effects associated with the pea-comb gene on chick weight and body weight and food efficiency of adult hens. Brit. Poult. Sci. 20, 463-472.

NEIIL A. R., REICHMANN K. G., ConNoR J. K. I977. Biochemical, physiological and production indices related to fat metabolism in the laying fowl ar various stages of physiological development. Brit. Poult. Sci., 18, 315-324.

RICARD F. H., I968. Essai d'estimation de l'épaisseur et de l'importance de la peau chez le poulet Ann. Zootech., 17, 459-466.

RICARD F. H., I972. Recherche d'une mesure précise de l'épaisseur de la peau chez le Poulet. Ann. Zootech., 21, 479-483.

RICARD F. H., I974. Étude de la variabilité génétique de quelques caractéristiques de carcasse en vue de sélectionner un poulet de qualité. C.R. I er Congrès mondial de génétique appliquée à la production animale, Madrid, 1, 93I-940.

RICARD F. H., 1977. Influence de l'âge et du patrimoine génétique sur l'état d'engraissement du poulet. In "La composition corporelle des volailles ", publ. Station de Recherches avicoles, I.N.R.A., p. $79-86$.

RICARD F. H., Rouvier R., I967. Étude de la composition anatomique du poulet de chair. I. Variabilité de la répartition des différentes parties corporelles chez des coquelets Bresse Pile. Ann. Zootech., 16, 23-39.

RICARD F. H., RoUviER R., I969. Eitude de la composition anatomique du poulet de chair. III. Variabilité de la répartition des parties corporelles dans une souche de type Cornish. Ann. Génét. Sél. anim. 1, I5I-I65.

WAShbURN K. W., GuilL R. A., EDwards H. M. Jr., I976. Influence of genetic differences in feed efficiency on carcass composition of young chicken. $J$. Nutr. 105, I311-1317.

WaTHES C. M., 1976. The effect of feathering on heat loss of the laying fowl. Gleadthorpe Poultry Booklet, 22-24. 